\title{
Effets combinés du compost, du Paraquat et de la Lambdacyhalothrine sur la macrofaune du sol sous culture pluviale de sésame (Sesamum indicum L.) au Burkina Faso
}

\author{
Paul W. SAVADOGO ${ }^{1 *}$, Yacouba ZI ${ }^{1}$, Abdoul K. SANOU ${ }^{1}$, Hassan B. NACRO ${ }^{2}$, \\ François LOMPO ${ }^{1}$ et Michel P. SEDOGO ${ }^{1}$ \\ ${ }^{1}$ Laboratoire Sol-Eau-Plante, Institut de l'Environnement et de Recherches Agricoles (INERA), \\ Ouagadougou, 01 BP 476 Ouagadougou 01, Burkina Faso. \\ ${ }^{2}$ Laboratoire d'Etude et de Recherche sur la Fertilité des Sols (LERF), Université Nazi Boni (UNB), \\ Bobo-Dioulasso, 01 BP. 1091 Bobo-Dioulasso 01, Burkina Faso. \\ *Auteur correspondant ; E-mail : paul.savadogo@gmail.com; Tel : +22670321619.
}

\section{RESUME}

Les pesticides chimiques de synthèse utilisés pour l'intensification des systèmes de production agricoles peuvent affecter de façon défavorable la qualité du sol à travers leur effet sur sa macrofaune. La présente étude a permis d'évaluer les effets synergiques du Paraquat et de la Lambdacyhalothrine en présence du compost sur la macrofaune du sol sous culture pluviale de sésame en milieu paysan, dans la région du Centre-Est du Burkina Faso. La méthode «Tropical Soil Biology and Fertility » (TSBF) a été utilisée pour évaluer l'abondance et la diversité de la macrofaune du sol après application de pesticides et de compost. L'étude a été menée entre 2014 et 2015 sur des monolithes de sols prélevés à des profondeurs de 0-10 $\mathrm{cm}$ et $10-30 \mathrm{~cm} 3$ jours avant semis, au $41^{\text {ème }}$ et au $81^{\text {ème }}$ jours après semis. Les résultats obtenus montrent qu'en absence de pesticide, le compost a entraîné une augmentation du nombre d'individus de la macrofaune du sol de $84,14 \%$ et de $173,46 \%$ respectivement dans les profondeurs de 0-10 cm et 10-30 cm du sol par rapport au témoin non traité. Il ressort aussi que le compost est plus favorable au développement équilibré et diversifié des groupes de macrofaune. Après traitement des parcelles par le Paraquat et la Lambdacyhalothrine, une diminution de 47,56\% de la macrofaune du sol a été notée à la profondeur de 0-10 cm du sol et leur élimination totale a été observée à la profondeur $10-30 \mathrm{~cm}$.

(C) 2017 International Formulae Group. All rights reserved.

Mots clés : Paraquat, Lambdacyhalothrine, Sesamum indicum L., macrofaune du sol.

\section{Combined effects of compost, Paraquat and Lambdacyhalothrine on soil macrofauna under rainfed crop of sesame (Sesamum indicum L.) in Burkina Faso}

\begin{abstract}
Synthesis chemical pesticides used for the intensification of agricultural production systems may affect adversely the quality of the soil through their effect on its macrofauna and its microflora. The present study aimed at evaluating the synergistic effects of Paraquat and Lambdacyhalothrin in the presence of the compost
\end{abstract}


on macrofauna of the soil under rainfed crop of sesame in peasant community, in the central east region of Burkina Faso. The method "Tropical Soil Biology and Fertility" (TSBF) has been used to assess the abundance and diversity of macrofauna of soil after application of pesticides and compost. To do so, monoliths of soils at depths of $0-10 \mathrm{~cm}$ and $10-30 \mathrm{~cm} 3$ days before seeding, at the $41^{\text {th }}$ and $81^{\text {th }}$ days after seedlings have been collected. The results showed that in the absence of pesticide, compost has led to an increase of the population of macrofauna in the soil by $84.14 \%$ and $173.46 \%$ respectively in the depths of $0-10 \mathrm{~cm}$ and $10-30 \mathrm{~cm}$ of the soil compared with the none treated sample. It is also clear that the compost is more favorable with the development of well-balanced and diversified groups of macrofauna. After treatment of plots by Paraquat and Lambdacyhalothrin, a decrease of $47.56 \%$ of the soil macrofauna has been recorded at the depth of $0-10 \mathrm{~cm}$ of the soil and their total elimination was observed at the depth of $10-30 \mathrm{~cm}$.

(C) 2017 International Formulae Group. All rights reserved.

Keywords: Paraquat, Lambdacyhalothrine, Sesamum indicum L., Soil macrofauna.

\section{INTRODUCTION}

$\mathrm{Au}$ Burkina Faso, le secteur agricole emploi plus de $86 \%$ de la population totale et génère près de 30 à $45 \%$ du produit intérieur brut (PIB) (MASA, 2014). La filière sésame est une composante majeure de ce secteur. Elle constitue l'une des principales sources de revenus pour $2 / 3$ des ménages agricoles (MAHRH, 2008). Les exportations de sésame sont devenues importantes avec une valeur de 45 milliards de FCFA en 2012 (Gildemacher et al., 2015). Cependant, ce secteur se trouve confronté aux attaques de pestes à différentes échelles, ce qui entraîne une baisse des rendements. Selon Rongead (2013), au Burkina Faso, les pertes en production causées par les maladies et les ravageurs se situent entre 20 et $25 \%$ des rendements. Ainsi, pour faire face à ces attaques parasitaires, nous assistons à une utilisation croissante des pesticides à des doses très variables (Gomgnimbou et al., 2009; Abang et al., 2014).

Bien que permettant de lutter efficacement contre les ravageurs des cultures, l'usage des pesticides peut causer des dommages entrainant un déséquilibre environnemental. En effet, plusieurs études ont montré que l'utilisation des pesticides entraîne une contamination des sols (Ondo et al., 2011), des eaux (Tapsoba et BonziCoulibaly, 2006), de l'air (Adu-Kumi et al.,
2012) et un risque pour la santé humaine et animale (Ahouangninou et al., 2011 ; Fianko et al., 2011; Bajwa, 2014). Après l'application des pesticides, une importante quantité se concentre dans le sol par le biais de processus de la bioaccumulation et de la bioamplification. Et selon Savadogo et al. (2009), cela peut porter préjudice aux organismes vivants du sol. En effet, des études ont révélé une diminution significative de l'activité enzymatique du sol suite à l'utilisation des pesticides tels que le Chlorpyrifos et le Quinalfos (Menon et al., 2005), le Chlorpyriphos-ethyl (Tejada et al., 2011) ainsi que de l'Endosulfan, la Deltamethrine et le Profenofos (Naré et al., 2014a). Cette activité est corrélée avec une baisse de la biomasse microbienne et de l'activité respiratoire du sol. L'étude menée par Charbonnier et al. (2015) a montré que l'application des pesticides réduit la densité de la macrofaune du sol. Pourtant, parmi les actions qui concourent à la protection du sol, un rôle prépondérant est attribué aux invertébrés du sol qui sont des indicateurs très sensibles de la qualité des sols et de leur fertilité (Lavelle et al., 2006; Ouédraogo et al., 2014). Il est donc nécessaire que ce patrimoine vivant du sol soit préservé.

L'amendement des sols par la matière organique, en particulier le compost, est pratiqué par la plupart des producteurs au 
Burkina Faso. Cette pratique, encouragée par les structures de recherche, apporte non seulement des éléments minéraux au sol mais aussi stimule l'activité des microorganismes du sol (Ros et al., 2003; Toundou et al., 2014). L'apport de la matière organique dans le sol se traduit également par une augmentation de la densité de sa macrofaune (Traore et al., 2012 ; Cluzeau et al., 2012; Naré et al., 2014b). Elle joue également un rôle important dans le devenir des pesticides (Savadogo et al., 2008 ; Tejada et al., 2011 ; Kumar et Singh, 2013).

Les études réalisées sur l'effet combiné des pesticides et du compost sur la macrofaune sont peu nombreuses en Afrique de l'Ouest et présentent des résultats parfois contradictoires. C'est dans ce contexte que s'inscrit la présente étude dont l'objectif était d'évaluer l'influence des pesticides sur la macrofaune du sol sous culture en présence de la matière organique.

\section{MATERIEL ET METHODES}

\section{Site d'étude}

Le site de l'étude se situe dans la commune de Bagré, région du Centre-Est du Burkina Faso, entre $11^{\circ} 32^{\prime} 51^{\prime \prime}$ de latitude Nord et $0^{\circ} 28^{\prime} 09^{\prime \prime}$ de longitude Ouest (Figure 1). Le climat y est tropical de type soudanien avec une saison de pluie allant de mai à octobre. La moyenne pluviométrique pour la campagne 2014-2015 est de $814 \mathrm{~mm}$. Les températures minima et maxima sont respectivement de 16,8 et $39,6{ }^{\circ} \mathrm{C}$. On y note des lithosols et des sols ferrugineux tropicaux lessivés (CETRI, 2014). Une analyse de ces sols donne les caractéristiques indiquées au Tableau 1.

\section{Pesticides utilisés}

Dans cette étude, deux pesticides ont été utilisés. Il s'agit du Supraxone, un herbicide de la famille chimique des
Bipyridylium avec pour principe actif le Paraquat (200 g/l) et la Lambda super 2.5 EC, un insecticide appartenant à la famille chimique des Pyrethrinoïdes avec pour principe actif la Lambdacyhalothrine $25 \mathrm{~g} / \mathrm{l}$. Le Paraquat a été appliqué avant le labour a raison de 4 1/ha. La Lambdacyhalothrine 2,5 EC a été appliquée à raison de $11 /$ ha. Elle a été apportée pour une première fois au $31^{\text {ème }}$ JAS correspondant au stade de la floraison. Une deuxième application a été faite 3 semaines après le premier traitement $\left(56^{\text {ème }}\right.$ JAS).

\section{Compost utilisé}

Le compost utilisé dont les caractéristiques sont consignées dans le Tableau 2 est produit par les agriculteurs. Il est fait à base de tiges de Andropogon gayanus, Pennisetum pedicellatum, Loudetia togoensis et apporté à la dose de 5 tonnes/ha.

\section{Matériel végétal}

Le sésame (Sesamum Indicum L.) est une plante annuelle de la famille des Pédaliacées cultivée dans les zones chaudes et moyennement humides. La plante a un développement de 0,70 à 2,30 mètres suivant les variétés (Rongead, 2013). Les graines de sésame sont logées dans des capsules à 2, 3 ou 4 lobes. La variété $\mathrm{S}-42$ a été utilisée dans le cadre de la présente étude. Cette variété a un cycle végétatif de 80 à 90 jours et son potentiel agronomique est de 1000 à 1500 $\mathrm{kg} / \mathrm{ha}$ de sésame graine en station de expérimentale. Les capsules sont déhiscentes à maturité. Le poids moyen de 100 graines se situe entre 3 et 5 grammes. Le taux de germination des semences est supérieur à 90 à $95 \%$ en première année et environ 70 à $75 \%$ en $3^{\text {ème }}$ année (Rongead, 2013). Les premières fleurs apparaissent après la $3^{\text {ème }}$ semaine et les capsules se forment à partir de la $4^{\text {ème }}$ ou $5^{\text {ème }}$ semaine. 


\section{Dispositif expérimental}

L'étude a été menée entre 2014 et 2015 et le dispositif expérimental était un bloc complètement randomisé comportant trois traitements et trois répétitions et placé sur un terrain à faible pente. D'une superficie totale de $868 \mathrm{~m}^{2}$ (35 m x 24,8 m), il comprenait trois blocs de 179,2 $\mathrm{m}^{2}$ (32 $\left.\mathrm{m} \times 5,6 \mathrm{~m}\right)$. Chaque bloc était constitué de trois parcelles élémentaires de $56 \mathrm{~m}^{2}$ (10 m x 5,6 m). L'allée entre deux blocs était de $2 \mathrm{~m}$ et les parcelles élémentaires étaient séparées entre elles par des allées de $1 \mathrm{~m}$. Les traitements étudiés étaient les suivants :

TNT : parcelle témoin non traité au pesticide et sans apport de compost ;

$\mathrm{C}$ : parcelle amendée au compost uniquement ;

$\mathrm{C}+\mathrm{P}$ : parcelle amendée au compost et traitée à l'herbicide Supraxone (Paraquat) avant semis puis à l'insecticide Lambdasuper (Lambdacyhalothrine) au $31^{\text {ème }}$ et $56^{\text {̀̀me }^{\text {jour }}}$ après semis du sésame.

Le semis a été effectué sur un sol labouré à plat avec un écartement de $20 \mathrm{~cm}$ entre les poquets et $60 \mathrm{~cm}$ entre les lignes.

\section{Etude de la macrofaune du sol}

L'échantillonnage de la macrofaune a été effectué 3 jours avant les semis ainsi qu'au $41^{\text {ème }}$ et au $81^{\text {ème }}$ jour après semis, par la méthode TSBF (Anderson et Ingram, 1993). Cette méthode consiste à prélever un monolithe de $25 \mathrm{~cm} \times 25 \mathrm{~cm} \times 30 \mathrm{~cm}$ de profondeur dans chaque parcelle. $\mathrm{Ce}$ monolithe de terre est d'abord subdivisé en deux strates $(0-10 \mathrm{~cm}$ et $10-30 \mathrm{~cm})$ ensuite fractionné et émietté à la main. Chacune des strates après concassage a été étalée sur une bâche en plastique et la macrofaune a été triée manuellement à l'aide de pinces fines. Les individus de la macrofaune ainsi récoltés sont conservés dans des bocaux contenant de l'alcool à $70 \%$. Les individus sont ensuite identifiés et dénombrés au laboratoire à l'aide d'une loupe binoculaire. Plusieurs clés de détermination ont été utilisées. Il s'agit de celle de Bland et Jacques (1978) pour la détermination des termites et des hémiptères ; Chinery (1981) pour les fourmis ; Bachelier (1978) pour les collemboles et les myriapodes et Csaba et Sandor (2010) pour les vers de terre.

\section{Traitement statistique des données}

Le logiciel Xlstat 2015 version 17.1.01 a été utilisé pour les analyses descriptives et de variances (ANOVA). La séparation des moyennes a été effectuée par le test de Student-Newman-Keuls au seuil de 5\%. La modélisation des graphiques a été réalisée à l'aide du logiciel Microsoft Excel 2016.

La diversité de la macrofaune du sol a été évaluée par des indices comme celui de Shannon-Weaver (H') et de l'Equitabilité (E). Les valeurs sont données par les formules suivantes : $\mathrm{H}^{\prime}=-\sum(\mathrm{Pi} \ln \mathrm{Pi}), \mathrm{Pi}$ étant la probabilité de rencontrer un taxon i sur une parcelle, se calcule de la façon suivante : $p(i)$ $=n i / N$ où $n i$ est le nombre d'individus pour l'espèce $i$ et $\mathrm{N}$ est l'effectif total des individus. Cet indice est accompagné par l'indice d'Equitabilité (E), qui traduit l'abondance relative des différentes espèces au sein du peuplement : $E=H^{\prime} / \ln (S)$, où $S$ est la richesse spécifique du peuplement (Magurran, 2006). L'abondance, un paramètre d'ordre quantitatif, a été utilisée pour exprimer le nombre d'individus d'une espèce par unité de surface. 


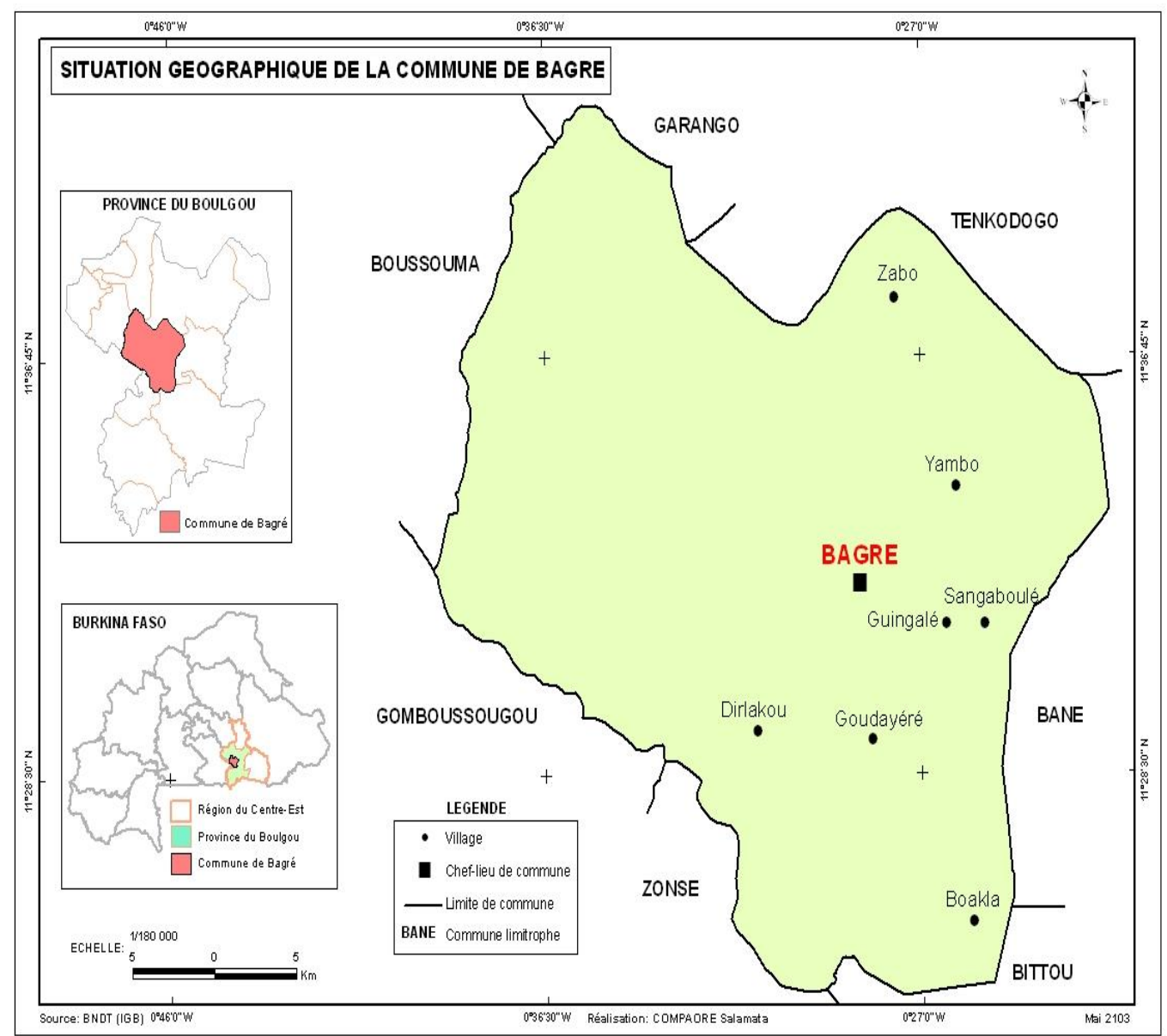

Figure 1 : Carte de la commune de Bagre, province du Boulgou, Burkina Faso.

Tableau 1 : Caractéristiques physico-chimiques des sols du site d'étude en fonction de la profondeur du sol.

\begin{tabular}{|c|c|c|c|c|c|c|c|c|c|c|c|c|c|}
\hline \multirow[b]{2}{*}{$\begin{array}{l}\text { Profondeur } \\
\text { Sol }(\mathrm{cm})\end{array}$} & \multicolumn{5}{|c|}{ Granulométrie (\%) } & \multirow[b]{2}{*}{$\begin{array}{c}\text { MO } \\
\text { total } \\
(\%)\end{array}$} & \multirow{2}{*}{$\begin{array}{c}\mathrm{C} \\
\text { total } \\
(\%)\end{array}$} & \multirow[b]{2}{*}{$\begin{array}{c}\mathrm{N} \\
\text { total } \\
(\%)\end{array}$} & \multirow[b]{2}{*}{$\mathbf{C} / \mathbf{N}$} & \multirow[b]{2}{*}{$\underset{(\mathbf{g} / \mathrm{kg})}{\mathbf{P}_{\text {ass }}}$} & \multirow[b]{2}{*}{$\begin{array}{c}\mathbf{K}_{\text {disp }} \\
(\mathrm{g} / \mathbf{k g})\end{array}$} & \multirow[b]{2}{*}{$\begin{array}{l}\text { pH } \\
\text { eau }\end{array}$} & \multirow[b]{2}{*}{$\begin{array}{l}\text { pH } \\
\text { KCL }\end{array}$} \\
\hline & Argile & $\begin{array}{l}\text { Limon } \\
\text { grossier }\end{array}$ & $\begin{array}{c}\text { Limon } \\
\text { fin }\end{array}$ & $\begin{array}{c}\text { Sable } \\
\text { grossier }\end{array}$ & $\begin{array}{c}\text { Sable } \\
\text { fin }\end{array}$ & & & & & & & & \\
\hline $0-20$ & 31,30 & 12,37 & 10,2 & 19,86 & 26,3 & 1,31 & 0,76 & 0,05 & 15,12 & 1,29 & 132,00 & 6,31 & 5,56 \\
\hline $20-40$ & 21,00 & 13,12 & 12,25 & 22,48 & 31,15 & 1,04 & 0,60 & 0,05 & 12,81 & 0,94 & 64,00 & 6,26 & 5,38 \\
\hline
\end{tabular}

Tableau 2 : Caractéristiques du compost utilisé.

\begin{tabular}{lccccccccc}
\hline Paramètres & $\begin{array}{c}\mathbf{p H} \\
\text { eau }\end{array}$ & $\begin{array}{c}\text { M O } \\
(\boldsymbol{\%})\end{array}$ & $\begin{array}{c}\mathbf{C} \text { total } \\
(\boldsymbol{\%})\end{array}$ & $\begin{array}{c}\mathbf{N} \text { total } \\
(\boldsymbol{\%})\end{array}$ & $\mathbf{C} / \mathbf{N}$ & $\begin{array}{c}\mathbf{P} \text { total } \\
(\mathbf{g} / \mathbf{k g})\end{array}$ & $\begin{array}{c}\mathbf{P} \text { ass } \\
(\mathbf{m g} / \mathbf{k g})\end{array}$ & $\begin{array}{c}\text { K total } \\
(\mathbf{g} / \mathbf{k g})\end{array}$ & $\begin{array}{c}\mathbf{K ~ d i s p} \\
(\mathbf{m g} / \mathbf{~ k g})\end{array}$ \\
\hline Valeurs & 7,46 & 66,5 & 38,57 & 2,4 & 16,07 & 0,65 & 103,67 & 3,51 & 153,06 \\
\hline
\end{tabular}

MO : matière organique ; $\mathrm{C}$ total : carbone total; $\mathrm{N}$ total : azote total ; Ptotal : phosphore total ; Pass : phosphore assimilable ; Ktotal : potassium total. Kdisp : potassium disponible. Source : ZI, 2016. 


\section{RESULTATS}

\section{Impact des traitements sur l'évolution de la macrofaune du sol}

Sur un total de 1065 individus de macrofaune identifiés, $64 \%$ de la densité totale proviennent de la profondeur $0-10 \mathrm{~cm}$ contre $36 \%$ à $10-30 \mathrm{~cm}$. La population de macrofaune est essentiellement constituée de Termites (Isoptères), de Fourmis (Hymenoptères), de vers de terre et autres (Myriapodes, Collemboles, Hémiptères) (Tableau 3).

La répartition des types de macrofaune diffère d'une période à l'autre et d'un traitement à l'autre (Figure 2). A $0-10 \mathrm{~cm}$ de profondeur du sol, nous constatons une augmentation significative de la macrofaune du sol dans le sol amendé au compost seul comparé au témoin non traité. Par contre, une diminution progressive de cette population de la macrofaune est observée dans le sol amendé au compost et traité au Paraquat et à la Lambdacyhalothrine. Ainsi, au $81^{\text {ème }}$ jour après semis, le compost a induit une augmentation de $84,14 \%$ de la densité totale de la macrofaune du sol alors que l'ajout de Lambdacyhalothrine et de Paraquat ont induit leur diminution de $47,56 \%$ comparé au témoin non traité.

Au niveau des profondeurs $10-30 \mathrm{~cm}$ $\mathrm{du}$ sol, les résultats montrent également une augmentation significative aux $41^{\text {ème }}$ et $81^{\text {ème }}$ jours après semis, de la macrofaune dans le sol ayant reçu uniquement le compost. Ce faisant, au $81^{\text {ème }}$ jour après semis, le compost a induit une augmentation de $173,46 \%$ de la densité totale de la macrofaune du sol par rapport au témoin non traité. Cependant, une diminution progressive jusqu'à une absence totale (au $81^{\text {ème }}$ jour après semis) de la macrofaune du sol est constaté dans le sol ayant reçu le compost et traité au Paraquat et à la Lambdacyhalothrine.

\section{Diversité résiduelle de la macrofaune du sol}

Les indices de Shannon-Weaver et d'Equitabilité évoluent dans le même sens entre 0 et $10 \mathrm{~cm}$ de profondeur du sol. Au $41^{\text {ème }}$ jour après semis, ils sont plus élevés dans le sol amendé au compost seul et faible dans le sol amendé au compost et traité aux pesticides (Figure 3 et Figure 4). Cela traduit une plus grande diversité de la macrofaune du sol dans la parcelle amendée au compost seul par rapport à celle amendée au compost et traitée aux pesticides. Toutefois, au $81^{\text {ème }}$ jour après semis, les valeurs des indices de Shannon-Weaver et d'Equitabilité sont obtenues aussi bien avec le sol amendé au compost seul qu'avec le sol amendé au compost et traité aux pesticides. Ce qui signifie qu'à cette période, l'effet de toxicité des pesticides a disparu et par conséquent, la diversité dans ces deux parcelles est rétablie.

A la profondeur de $10-30 \mathrm{~cm}$, le sol amendé au compost seul et le sol amendé au compost puis traité aux pesticides ont enregistré des indices de Shannon et d'Equitabilité plus élevés au $41^{\text {ème }}$ jour après semis par rapport au témoin non traité (Figures $3 b$ et $4 b$ ). Cela indique que ces parcelles présentent une population de la macrofaune du sol plus diversifiée comparé au témoin. 
Tableau 3 : Individus et nombre de macrofaune du sol à 0-10 $\mathrm{cm}$ et $10-30 \mathrm{~cm}$ de profondeur.

\begin{tabular}{|c|c|c|c|c|}
\hline $\begin{array}{c}\text { Période de } \\
\text { prélèvement du sol }\end{array}$ & Traitements & $\begin{array}{c}\text { Type de } \\
\text { macrofaune }\end{array}$ & $\begin{array}{l}\text { Nombre/m² } \\
(0-10 \mathrm{~cm})\end{array}$ & $\begin{array}{l}\text { Nombre/m² } \\
(10-30 \mathrm{~cm})\end{array}$ \\
\hline \multirow{4}{*}{3 jours avant semis } & \multirow{4}{*}{$\begin{array}{l}\text { Sol sans apport de } \\
\text { compost et pesticide }\end{array}$} & Vers de terre & $32 \pm 3,46$ & $11 \pm 2,31$ \\
\hline & & Termites & $38 \pm 3,60$ & $43 \pm 3,05$ \\
\hline & & Fourmis & $16 \pm 1,73$ & $0 \pm 0,00$ \\
\hline & & Autres & $21 \pm 2,20$ & $11 \pm 2,31$ \\
\hline \multirow{12}{*}{$\mathrm{Au} 41^{\text {ème }} \mathrm{JAS}$} & \multirow{4}{*}{ Témoin non traité } & Vers de terre & $0 \pm 0,00$ & $0 \pm 0,00$ \\
\hline & & Termites & $43 \pm 4,36$ & $32 \pm 3,46$ \\
\hline & & Fourmis & $32 \pm 4,00$ & $38 \pm 4,16$ \\
\hline & & Autres & $0 \pm 0,00$ & $0 \pm 0,00$ \\
\hline & \multirow{4}{*}{$\begin{array}{c}\text { Sol amendé au Compost } \\
\text { Uniquement }\end{array}$} & Vers de terre & $11 \pm 2,31$ & $6 \pm 1,53$ \\
\hline & & Termites & $70 \pm 9,24$ & $32 \pm 4,00$ \\
\hline & & Fourmis & $43 \pm 4,62$ & $54 \pm 5,03$ \\
\hline & & Autres & $0 \pm 0,00$ & $0 \pm 0,00$ \\
\hline & \multirow{4}{*}{$\begin{array}{l}\text { Sol amendé au Compost et } \\
\text { traité aux pesticides }\end{array}$} & Vers de terre & $0 \pm 0,00$ & $6 \pm 0,57$ \\
\hline & & Termites & $70 \pm 7,02$ & $54 \pm 5,03$ \\
\hline & & Fourmis & $27 \pm 4,16$ & $0 \pm 0,00$ \\
\hline & & Autres & $0 \pm 0,00$ & $16 \pm 4,00$ \\
\hline \multirow{12}{*}{$\mathrm{Au} 81^{\text {ème }} \mathrm{JAS}$} & \multirow{4}{*}{ Témoin non traité } & Vers de terre & $0 \pm 0,00$ & $0 \pm 0,00$ \\
\hline & & Termites & $70 \pm 9,45$ & $43 \pm 4,62$ \\
\hline & & Fourmis & $6 \pm 0,58$ & $0 \pm 0,00$ \\
\hline & & Autres & $6 \pm 0,58$ & $6 \pm 0,58$ \\
\hline & \multirow{4}{*}{$\begin{array}{c}\text { Sol amendé au Compost } \\
\text { Uniquement }\end{array}$} & Vers de terre & $0 \pm 0,00$ & $0 \pm 0,00$ \\
\hline & & Termites & $91 \pm 8,33$ & $112 \pm 12,17$ \\
\hline & & Fourmis & $38 \pm 4,62$ & $16 \pm 4,00$ \\
\hline & & Autres & $22 \pm 2,31$ & $6 \pm 0,58$ \\
\hline & \multirow{4}{*}{$\begin{array}{l}\text { Sol amendé au Compost et } \\
\text { traité aux pesticides }\end{array}$} & Vers de terre & $0 \pm 0,00$ & $0 \pm 0,00$ \\
\hline & & Termites & $11 \pm 2,30$ & $0 \pm 0,00$ \\
\hline & & Fourmis & $32 \pm 3,46$ & $0 \pm 0,00$ \\
\hline & & Autres & $0 \pm 0,00$ & $0 \pm 0,00$ \\
\hline
\end{tabular}




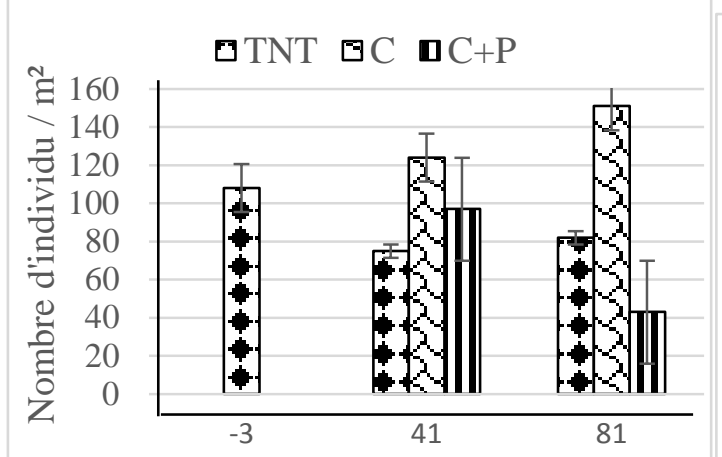

$\mathbf{a}$

Nombre de jours après semis

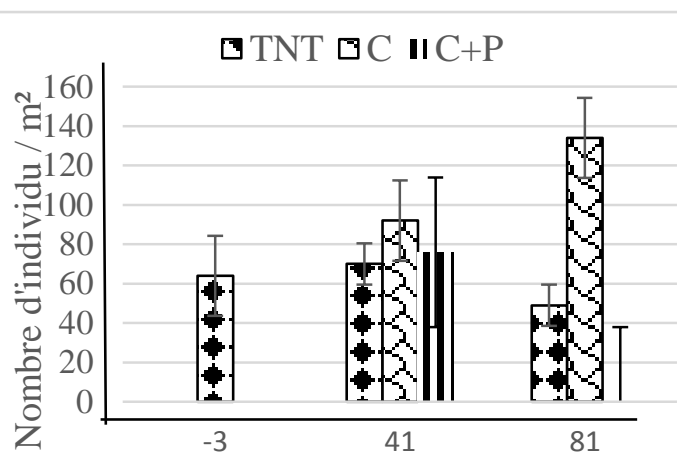

b Nombre de jours après semis

TNT = Témoin non traité, $\mathrm{C}=\mathrm{Sol}$ amendé au Compost uniquement, $\mathrm{C}+\mathrm{P}=\mathrm{Sol}$ amendé au Compost et traité aux pesticides

Figure 2 : Evolution de la densité moyenne de la macrofaune du sol à : a) $0-10 \mathrm{~cm}$ et b) $10-30$ $\mathrm{cm}$ de profondeur du sol en fonction des traitements.

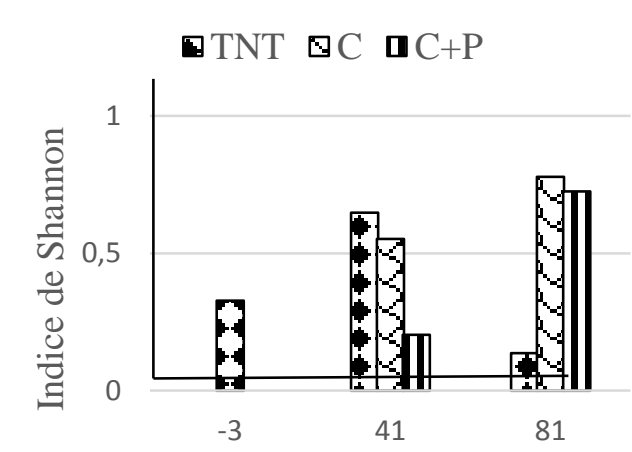

a) Nombre de jours après semis

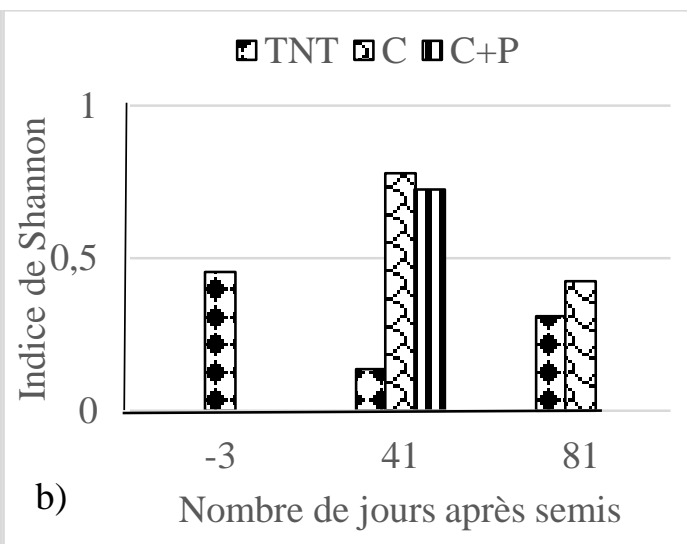

b) Nombre de jours après semis

TNT = Témoin non traité, $\mathrm{C}=\mathrm{Sol}$ amendé au Compost uniquement, $\mathrm{C}+\mathrm{P}=\mathrm{Sol}$ amendé au Compost et traité aux pesticides

Figure 3 : Indice de Shannon à : a) $0-10 \mathrm{~cm}$ et b) $10-30 \mathrm{~cm}$ de profondeur du sol en fonction des traitements.

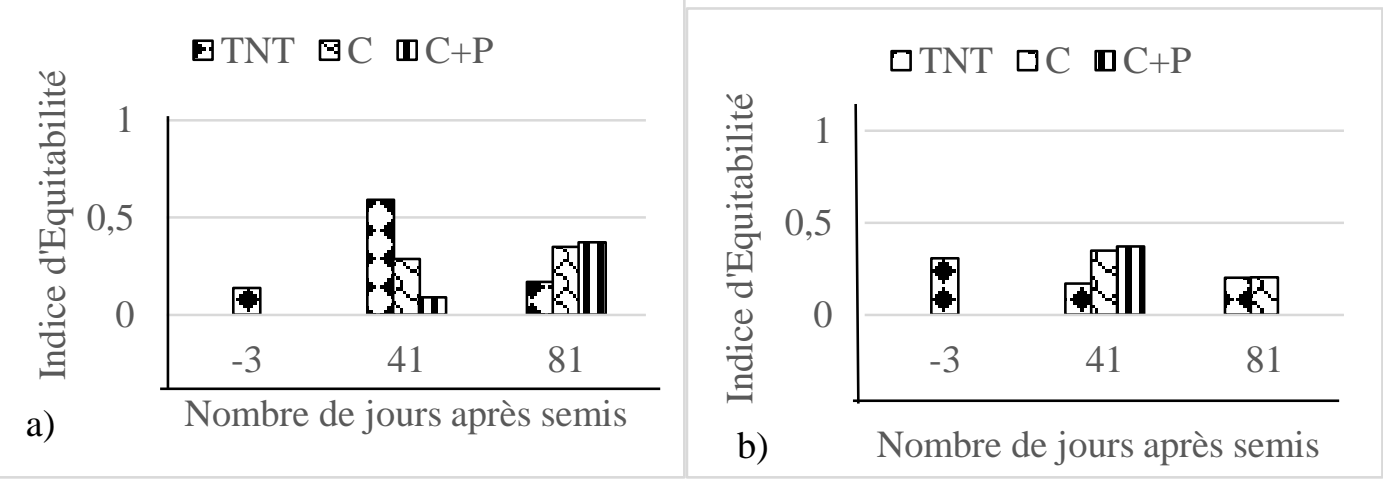

Figure 4 : Indice d'Equitabilité à : a) $0-10 \mathrm{~cm}$ et b) $10-30 \mathrm{~cm}$ de profondeur du sol en fonction des traitements. $\mathrm{TNT}=$ Témoin non traité, $\mathrm{C}=\mathrm{Sol}$ amendé au Compost uniquement, $\mathrm{C}+\mathrm{P}=\mathrm{Sol}$ amendé au Compost et traité aux pesticides. 


\section{DISCUSSION}

L'apport du compost s'est traduit par une augmentation progressive au cours du temps, de la population de termites, de fourmis, de vers de terre, de myriapodes, de collemboles et d'hémiptères aussi bien dans les profondeurs 0-10 $\mathrm{cm}$ que $10-30 \mathrm{~cm}$ du sol. Cet effet pourrait s'expliquer par le fait que l'apport du compost, fourni une source d'éléments nutritifs aux organismes présents dans le sol, entraînant ainsi leur croissance et leur développement (Charland et al., 2001). Nos résultats sont conformes à ceux obtenus par d'autres auteurs (Traore et al., 2012 ; Cluzeau et al., 2012; Naré et al., 2014b; Mbau et al., 2015). En effet, l'étude de Naré et al. (2014b) a révélé une augmentation de $69,66 \%$ la densité totale de la macrofaune du sol due à l'apport de la matière organique comparativement aux parcelles sans amendement organique. Par ailleurs, l'étude menée par Mbau et al. (2015) au Kenya a mis en évidence une augmentation du nombre de termites, de vers de terres et de fourmis par unité de surface, respectivement de 44\%, 26\% et $17 \%$. De même, les travaux de Traoré et al. (2012) réalisés au Burkina Faso ont montré que le traitement par des résidus de paille entraînait aussi bien une augmentation du nombre d'individus que la diversité de la macrofaune telluriques.

Nos résultats ont montré une diminution de la macrofaune dans les parcelles amendées au compost et traitées aux pesticides comparé au sol amendé au compost seul et au témoin non traité. Cette baisse peut être attribuée à l'effet combiné de la toxicité du Paraquat et de la Lambdacyhalothrine sur la macrofaune. Ce qui montre que les pesticides étudiés ont été toxiques pour la macrofaune du sol entraînant ainsi une baisse de sa densité. Nos résultats corroborent ceux de plusieurs auteurs dont Robertson et al. (1998), Charbonnier et al. (2015) et Naré et al. (2017). Ces derniers auteurs, dans une étude similaire, ont montré que la Lambdacyhalothrine entraînait une élimination totale de la population de termites même en présence du compost. Ils ont aussi montré que le Chlorpyrifos entraînait une diminution de la densité de la population de fourmis, de coléoptères, d'hémiptères, de collemboles, de myriapodes, d'arachnides et de diptères. De même, les travaux menés par Vavoulidou et al. (2009) sont en faveur d'une élimination totale de la macrofaune du sol due à l'application d'un insecticide organophosphoré nommé « Cadusaphos ».

Les parcelles amendées au compost présentent le meilleur indice de diversité et d'équitabilité de la macrofaune du sol. Cela montre que le compost entraine un développement équilibré et diversifié des groupes de macrofaune. En effet, le compost inocule dans le sol des milliards d'organismes vivants et diversifiés, accroissant ainsi sa biodiversité (Charland et al., 2001 ; Förster et al. 2006). Par ailleurs, le compost favorise également le développement de la macrofaune du sol dû aux nutriments divers qu'il renferme (Charland et al., 2001). Nos résultats concernant l'impact des pesticides testés sur la diversité de la macrofaune corroborent ceux de Traoré et al. (2012) et Naré et al. (2017) qui ont obtenu un meilleur indice de diversité et d'équitabilité de la macrofaune du sol en présence du compost. Ainsi, le compost améliore la diversité de la population de macrofaune, toute chose qui est en faveur d'une fertilisation des sols par application du compost. Cependant, la diversité de la macrofaune du sol au $81^{\text {ème }}$ jour après semis dans le sol amendé au compost et traité aux pesticides à la profondeur de $0-10 \mathrm{~cm}$ pourrait s'expliquer par une remonté des termites pour se nourrir de la biomasse sèche en surface qui se constitue en fin de saison pluvieuse. Nos résultats corroborent ceux de Traoré et al. (2015) qui ont montré que les termites présenteraient une meilleure adaptation dans un milieu mis en culture et surtout lorsque le sol est enherbé. De même, Ouédraogo et al. (2015) ont montré que l'importance des dégâts par les termites sur les cultures est observée durant les poches de sécheresse. Ainsi, cette remontée des termites entraînerait une arrivée massive des fourmis qui les consomment. 


\section{Conclusion}

Les effets de l'utilisation des pesticides et du compost sur la macrofaune du sol ont été étudiés au cours de ce travail. Notre étude a montré que l'apport du compost s'est traduit par une stimulation de la population de la macrofaune du sol et une augmentation de sa diversité. Les résultats ont par ailleurs montré que l'application de la Lambdacyhalothrine et du Paraquat a entraîné une diminution de la macrofaune du sol, même en présence du compost. Par conséquent, nous pouvons conclure que l'effet bénéfique du compost peut être inhibé par les effets combinés du Paraquat et de la Lambdacyhalothrine. Ainsi, dans un contexte de dégradation continue du sol, l'amendement organique est conseillé pour la gestion de la fertilité du sol. Toutefois, les traitements du sol par les pesticides devront être évités dans la mesure du possible, afin de maintenir la densité et la diversité de la macrofaune et garantir les bénéfices du compost pour la fertilisation de sol et la promotion d'une agriculture durable.

\section{CONFLITS D'INTERETS}

Les auteurs déclarent qu'ils n'ont aucun conflit d'intérêts.

\section{CONTRIBUTIONS DES AUTEURS}

PWS a rédigé le projet de recherche ainsi que le premier draft de l'article et l'a soumis aux co-auteurs et au journal. Il était l'encadreur de $\mathrm{YZ}$ au laboratoire à l'INERA. YZ a effectué son stage de Master 2 sur le site et a collecté les résultats aussi bien en milieu réel qu'au laboratoire sous la supervision de AKS. HBN, FL et MPS sont respectivement le superviseur de YZ à l'Université, le coordonnateur du laboratoire à l'INERA et le chef de l'équipe scientifique de recherche. Ils ont tous fortement contribué à la rédaction de l'article.

\section{REMERCIEMENTS}

Nos vifs remerciements à Maurice Ouango SAVADOGO pour son aide lors de l'analyse statistique des données.

\section{REFERENCES}

Abang AF, Kouamé CM, Abang M, Hanna R, Fotso AK. 2014. Assessing Vegetable Farmer Knowledge of Diseases and Insect Pests of Vegetable and Management Practices Under Tropical Conditions. Int. J. Veg. Sci., 20: 240253. DOI: $10.1080 / 19315260.2013$. 800625

Adu-Kumi S, Kareš R, Literák J, Boru vková J, Yeboah PO, Carboo D, Akoto O, Darko G, Osae S, Klánová J, 2012. Levels and seasonal variations of organochlorine pesticides in urban and rural background air of southern Ghana. Environmental Science and Pollution Research, 19(6):1963-1970. DOI: 10.1017/s11356-012-1013-y

Ahouangninou C, Fayomi BE, Martin T. 2011. Evaluation des Risques Sanitaires et Environnementaux des Pratiques Phytosanitaires des Producteurs Maraîchers dans la Commune Rurale de Tori-Bossito (Sud-Bénin). Cahiers Agricultures, 20(3): 216-222. DOI: http://dx.doi.org/10.1684/agr.2011.0485

Bachelier G. 1978. La Faune des Sols : son Ecologie et son Action. ORSTOM: Paris, France; 400p.https:horizon. documentation.ird.fr/exl-doc/pleins_ textes/pleins_textes_6/.../09691.pdf

Bajwa U, Sandhu KS. 2014. Effect of handling and processing on pesticide residues in food - a review. Journal of Food Science and Technology, 51: 201220. DOI: $10.1007 / \mathrm{s} 13197-011-0499-5$

Bland RG, Jaques HE. 1978. How to Know the Insects, The pictured Key Nature Series $\left(3^{\text {rd }}\right.$ edn), Brown, Dubuque: Lowa. 409.

CETRI. 2014. Projet de plantation de 220 hectares de Moringa oleifera associé à d'autres cultures en goutte à goutte à Bagré, Rapport de l'étude d'impact 
environnemental et social, Fondation Dreyer, Dano, Burkina Faso. 150p.

Charbonnier E, Ronceux A, Carpentier AS, Soubelet H, Barriuso E. 2015 : Pesticides : des Impacts aux Changements de Pratiques. Bilan de Quinze Années de Recherche pour Eclairer la Décision Publique. Editions Quae : Versailles Cedex, France ; 400.

Charland M, Cantin S, St-Pierre MA, Côté L. 2001. Recherche sur les avantages à utiliser le compost. Centre de recherche industrielle du Québec (CIRQ), RECYCQUEBEC PE27158(R1), 49p.

Chinery M. 1981. Le Multiguide Nature des Insectes d'Europe en Couleur. Bordas: Paris, France ; 380.

Cluzeau D, Guernion M, Chaussod R, MartinLaurent F, Villenav C. 2012. Integration of biodiversity in soil quality monitoring: Baselines for microbial and soil fauna parameters for different landuse types. European Journal of Soil Biology, 49: 63-72. DOI:10.1016/j.ejsobi.2011.11.003

Csaba C, Sandor M. 2010. A Monograph of the Paleotropical Benhamiinae earthworms (Annelida: Oligochaeta, Acanthodrilidae). Edition: Hungarian Natural History Museum and Systematic Zoology Research Group of the Hungarian Academy of Sciences: Hongrie, Budapest; 348.

Fianko JR, Donkor A, Lowor ST, Yeboah PO, Glover ET, Adom T, Faanu A. 2011. Health Risk Associated with Pesticide Contamination of Fish from the Densu River Basin in Ghana. Journal of Environmental Protection, 2: 115-123. DOI: 10.4236/jep.2011.22013

Förster B, Garcia M, Francimari O, Römbke J. 2006. Effects of carbendazim and lambda cyhalothrin on soil invertebrates and leaf litter decomposition in semifield and field tests under tropical conditions (Amazônia, Brazil). European Journal of Soil Biology, 42(1): S171S179. DOI: 10.1016/j.ejsobi.2006.07.011 Gildemacher P, Audet-Bélanger G, Mangnus E, Van de Pol F, Tiombiano D, Sanogo K. 2015. Développement de la Filière Sésame; Capitalisation des Acquis au Burkina Faso et au Mali. KIT \& CFC: Amsterdam, 48p.

Gomgnimbou APK, Savadogo PW, Nianogo AJ, Millogo-Rasolodimby J. 2009. Usage des intrants chimiques dans un agrosystème tropical : diagnostic du risque de pollution environnementale dans la région cotonnière de l'est du Burkina Faso. Biotechnol. Agron. Soc. Environ, 13(4): 499-507. http://popups.ulg.ac.be/17804507/index.php?id=4524.

Kumar A, Singh S. 2013. Adsorption and Desorption Behavior of Chlorotriazine Herbicides in the Agricultural Soils. $J$. Pet. Environ. Biotechnol., 4(5): 154. DOI: 10.4172/2157-7463.1000154

Lavelle P, Decaens M, Aubert M, Barot S, Bloiun M, Bureau F, Magerie P, Mora P, Rossi JP. 2006. Soil invertebrates and ecosystems services. European Journal of Soil Biology, 42(1): 3-15. https://doi.org/10.1016/j.ejsobi.2006.10. 002

MAHRH (Ministère de l'agriculture de l'hydraulique et des ressources halieutiques), 2008. Rapport de prédiagnostic de la filière sésame. Burkina Faso. 20p. https : doczz.fr/doc/2589640/la-filière-sésameau-Burkina-Faso

MASA (Ministère de l'agriculture et de la sécurité alimentaire), 2014. Résultats définitifs de la campagne agricole et de la situation alimentaire et nutritionnelle 2013/2014. Burkina Faso. 77p. https:www.cns.bf/IMG/pdf/rapport_gene ral_cpsa_2014.pdf 
Mbau SK, Karanja N, Ayuke F. 2015. Shortterm influence of compost application on maize yield, soil macrofauna diversity and abundance in nutrient deficient soils of Kakamega County, Kenya. Plant and Soil; 1-2(387): 379-394. DOI: 10.1007/s11104-014-2305-4

Menon P, Gopal M, Parsad R. 2005. Effects of chlorpyriphos and quinalphos on deshydrogenase activities and reduction of $\mathrm{Fe}^{3+}$ in the soil of two semi-arid fields of tropical Indian. Agronomy, Economy and Environment, 108: 73-83. DOI: 10.1016/j.agee.2004.12.008

Naré RWA, Gountan A, Traoré M, Savadogo PW, Nacro HB. and Sedogo MP. 2014b. Effect of pesticides and organic amendments on soil macrofauna and microbial respiration activities under rainfed tomato (Solanum lycopersicum L.). Agroecology for Africa, 1-5. https: www.cirad.mg/conference/AfA2014/?dl_id=119

Naré RWA, Savadogo PW, Gnankambary Z, Nacro HB and Sedogo PM. 2014a. Effect of Three Pesticides on Soil Dehydrogenase and Fluorescein Diacetate Activities in Vegetable Garden in Burkina Faso. Curr. Res. J. Biol. Sci., 6(2): 102-106.

Naré RWA, Savadogo PW, Traore M, Gountan A, Nacro HB and Sedogo MP. 2017. Soil Macrofauna Behaviour in the Presence of Pesticides and Organic Amendments. Journal of Geoscience and Environment Protection, 5: 202-212. DOI: 10.4236/gep.2017.53014

Ondo Z, Alibert P, Dousset S, Savadogo PW, Savadogo M, Sedogo M. 2011. Insecticide residues in cotton soils of Burkina Faso and effects of insecticides on fluctuating asymmetry in honey bees (Apis mellifera Linnaeus). Chemosphere; 83: 585-592. DOI: 10.1016/j.chemosphere.2010.12.021
Ouédraogo I, Hema SO, Traore M, Sanon F. 2015. Diversité des termites et leur Impacts dans les champs de maïs dans deux zones agro-écologiques au Burkina Faso. Journal of Animal \&Plant Sciences, $\quad 3(26)$ : $\quad 4150-4160$. http://www.m.elewa.org/JAPS.

Ouédraogo J, Nacro HB, Ouédraogo E, Youl S, Sedogo MP. 2014. Amélioration de la disponibilité du phosphore par la gestion de la macrofaune du sol: cas d'un lixisol en zone semi-aride du Burkina Faso. Int. J. Biol. Chem. Sci., 8(4): 18381846. DOI: http://dx.doi.org/10.4314/ ijbcs.v8i4.41

Robertson LN, Chandler KJ, Stickley BDA, Cocco RF, Ahmetagic M. 1998. Enhanced microbial degradation implicated in rapid loss of chlorpyrifos from the controlled release formulation susucon(R) Blue in soil. Crop Protection, 17: 29-33. https://doi.org/10.1016/S02612194(98)80009-4

Rongead. 2013. Le sésame au Burkina Faso : état des lieux 2013, Burkina Faso, livret 3 ; 16p. http://www.rongead.org/ OUTILS-PEDAGOGIQUESBURKINA-FASO.html

Ros M, Hernandez MT, Garcia C. 2003. Soil microbial activity after restoration of a semi arid soil by organic amendments. Soil Biol. Biochem, 3(35): 463-469. DOI: 10.1016/S0038-0717(02)00298-5

Savadogo PW, Lompo F, Bonzi-Coulibaly YL, Traoré AS, Sedogo PM. 2008. Influence de la Température et des Apports de Matière Organique sur la Dégradation de l'Endosulfan dans trois types de Sols de la Zone Cotonnière du Burkina Faso. Journal de Societé OuestAfricaine de Chimie, 026: 79 - 87. http://docplayer.fr/45087272-J-socouest-afr-chim-2008-026-79-87.html 
Savadogo PW, Lompo F, Coulibaly K, Traoré O, Traoré AS, Sedogo PM. 2009. A microcosm Study of Endosulfan Degradation and its Short-Term Effect on $\mathrm{pH}$ and Biological Parameters of Cotton Zones Soils of Burkina Faso. Journal of Environment Science and Technology, 2(1): 12-21. DOI: 10.3923/jest.2009.12.21

Tapsoba HK, Bonzi-Coulibaly YL. 2006. Production cotonnière et pollution des eaux par les pesticides au Burkina Faso. Journal de Société Ouest-Africaine de Chimie, 21 :87-93.

Tejada M, Gòmez I, Del Toro M. 2011. Use of organic amendments as a bioremediation strategy to reduce the bioavailability of chlorpyrifos insecticide in soils. Effects on soil biology. Ecotoxicology and Environmental Safety, 74: 2075-2081. DOI: 10.1016/j.ecoenv.2011.07.005

Toundou O, Tozo K, Feuillade G, Pallier V, Tchegueni S, Dossou KSS. 2014. Effets de composts de déchets sur les propriétés chimiques du sol et la solubilité d'éléments minéraux sous deux régimes hydriques en conditions contrôlées au Togo. Int. J. Biol. Chem. Sci, 8(4): 19171926. DOI : http://dx.doi.org/10.4314/ ijbcs.v8i4.51

Traore M, Lompo F, Ayuke F, Ouattara B, Ouattara K, Sedogo PM. 2012. Influence des pratiques agricoles sur la macrofaune du sol : cas de l'enfouissement de la paille et du fumier. Int. J. Biol. Chem. Sci., 6(4): 1761-1773. DOI : http://dx.doi.org/10.4314/ijbcs.v6i4.31

Traore M, Barro A, Garane A et Nacro HB. 2015. Etude de la dynamique de la macrofaune du sol sous culture de deux variétés de patate douce (Ipomea batatas) avec utilisation de différents modes de désherbage. Int. J. Biol. Chem. Sci., 9(5): 2334-2345. DOI : http://dx.doi.org/10.4314/ijbcs.v9i5.7

Vavoulidou E, Avramides E, Wood M, Lolos P. 2009. Response of Soil Quality Indicators to the Pesticide Cadusaphos. Communications In Soil Science And Plant Analysis, 1-6(40): 419-434. DOI: http://dx.doi.org/10.1080/001036208026 97996

Zi Y. 2016. Effet des pesticides et du compost sur les paramètres physico-chimiques et biologiques du sol sous culture pluviale de sésame (Sesamum indicum L.) dans la région du Centre-Est du Burkina Faso : cas de la commune de Bagré. Mémoire d'Ingénieur du Développement Rural, option Agronomie. Université Polytechnique de Bobo, Institut du Développement Rural, Bobo-Dioulasso, Burkina Faso, 78p. 